\title{
Electrical resistivity and structural changes upon relaxation and crystallisation of $\left(\mathrm{Mo}_{0.6} \mathrm{Ru}_{0.4}\right)_{100-x} \mathrm{~B}_{x}$ metallic glasses
}

\author{
Robert Schulzt, Madhav Mehra and W L Johnson \\ W M Keck Laboratory of Engineering Materials. California Institute of Technology. \\ Pasadena, California 91125, USA
}

Received 5 June 1984, in final form 6 March 1985

\begin{abstract}
Crystallisation of $\left(\mathrm{Mo}_{0.6} \mathrm{RL}_{0.4}\right)_{100-x} \mathrm{~B}_{x}$ glasses takes place in three steps: the first step corresponds to the precipitation of the sigma phase $\mathrm{Mo}_{5} \mathrm{Ru}_{3}$ which decomposes at higher temperatures, the second step is associated with the formation of an HCP solid solution of Mo in $\mathrm{Ru}$, and in the last step the remaining amorphous matrix crystallises in an FCC boride.

Detailed electrical resistivity measurements taken below the crystallisation temperature reveal an excess resistivity above the usual linear temperature dependence predicted by the Ziman theory. The increase in resistivity is associated with the onset of long-range compositional inhomogeneity (spinodal decomposition) and the decrease with the onset of crystallisation. The electrical behaviour for samples pre-annealed for 12 hours at various temperatures suggests that boron migration is partly responsible for this excess resistivity. The changes in the radial distribution function of $\left(\mathrm{Mo}_{0.6} \mathrm{Ru}_{0.4}\right)_{78} \mathrm{~B}_{22}$ during annealing at $525^{\circ} \mathrm{C}$ are also reported.

The Mott $s-d$ scattering model does not seem to be applicable to these amorphous alloys. The data suggest instead that an important contribution to the conduction comes from the d electrons.
\end{abstract}

\section{Introduction}

It is well known that electrical resistivity measurements form a sensitive probe for the experimental investigation of relaxation and crystallisation processes in metallic glasses. Both topological (free-volume annihilation) and chemical relaxation (ordering, phase separation ...) influence the electrical resistivity. The problem arises in the interpretation of the data, in other words when we try to assign a specific mechanism or structural transformation to a particular feature in the electrical resistivity.

Reversible changes are usually considered as chemical ordering [1] and irreversible changes as topological relaxation [2]; the latter have been studied extensively. Allia et al [3] used a theory based on the Ziman approach to the resistivity and the microscopic model for structural relaxation proposed by Srolovitz et al [4] to explain the free-volume dependence of the electrical resistivity. They attributed the increase of resistivity in their low-temperature isothermal measurements to changes in compositional short-range order (clustering of Mo atoms in the case of $\mathrm{Fe}-\mathrm{Ni}-\mathrm{Mo}-\mathrm{B}$ alloys for instance) and the decrease at high temperatures to topological relaxation [5]. Their results, however, are in

† Present address: General Motors Research Laboratories. Physical Chemistry Department. Warren. Michigan 48090-9055, USA. 
disagreement with those of Venkataraman et al [6]. Kelton and Spaepen [7], on the other hand, assign the increase as well as the decrease of the resistivity in $\mathrm{Pd}-\mathrm{Si}-\mathrm{V}$ alloys to free-volume relaxation and not to the clustering of vanadium atoms. The reason for many of these disagreements is the lack of direct structural information correlated with the resistivity measurements.

The effect of chemical relaxation on the electrical transport in metallic glasses is poorly understood. Even when atomic clustering and phase separation [8-10] had been observed in metallic glasses, their effects on the electrical resistivity were never carefully investigated in the amorphous state until recently [11]. In the crystalline state, however, the behaviour of the resistivity during Guinier-Preston zone formation, spinodal decomposition, etc, is well known [12-15].

Considerable work has already been done on the effect of annealing on the structure of $\left(\mathrm{Mo}_{0.6} \mathrm{Ru}_{0.4}\right)_{100-x} \mathrm{~B}_{x}$ metallic glasses [16-18]. It has been suggested that upon annealing at temperatures sufficiently high to permit the diffusion of boron over atomic distances, but low enough to prevent crystallisation, this glass phase separates into boron-rich and boronpoor zones. Thus Mo-Ru-B represents an ideal candidate to study the effect of chemical segregation on the electrical properties of this glass. This paper reports electrical resistivity and high-angle $\mathrm{x}$-ray diffraction measurements upon annealing $\left(\mathrm{Mo}_{0.6} \mathrm{Ru}_{0.4}\right)_{100-x} \mathrm{~B}_{x}$ for $x=14,18$ and 22 and interprets the results in terms of phase separation caused by diffusion of boron in the amorphous matrix.

\section{Experiment}

The ingots were prepared by RF-induction-melting the different constituents on a silver boat in an argon atmosphere. The ingots were remelted several times to ensure homogeneity. The rapidly quenched amorphous alloys were obtained by the "piton and anvil' technique [19]. The foils were typically $40 \mu \mathrm{m}$ in thickness and 1.5 to $2 \mathrm{~cm}$ in diameter. They were examined by a high-angle $\mathrm{x}$-ray diffractometer using $\mathrm{Cu} \mathrm{K}_{\alpha}$ radiation and only the foils showing a broad band with no resolvable Bragg peaks were kept for further study. The detailed diffraction studies of the crystalline phases obtained from the amorphous samples by heating through the crystallisation temperature were performed on the same $\mathrm{x}$-ray Norelco diffractometer by scanning at $0.05^{\circ}$ intervals. The $\mathrm{x}$-ray data used in computing the radial distribution functions (RDF) were obtained in the transmission geometry on a Norelco scanning goniometer using Mo $\mathrm{K}_{\alpha}$ radiation and a focusing LiF monochromator. The details of the data handling and RDF calculation were described elsewhere $[20,21]$. The resistivity was measured using a four-point $\mathrm{DC}$ method with $\mathrm{Pt}$ leads spot welded on to the samples, also described elsewhere [11].

\section{Results and discussion}

There is now much evidence to believe that alloys with low boron content $(x<18)$ have predominantly one type of local structure while those with high boron content $(x>18)$ have a distinct second type of local structure $[17,22,23]$. We therefore expect different crystallisation behaviour as we go through the concentration range. Figure 1 shows the normalised electrical resistivity for $x=14,18$ and 22 as we heat the samples through the crystallisation temperature at a heating rate of about $15^{\circ} \mathrm{C} \mathrm{min}-1$. For the low boron concentration alloys we find a three-step process, the first two becoming almost a single 


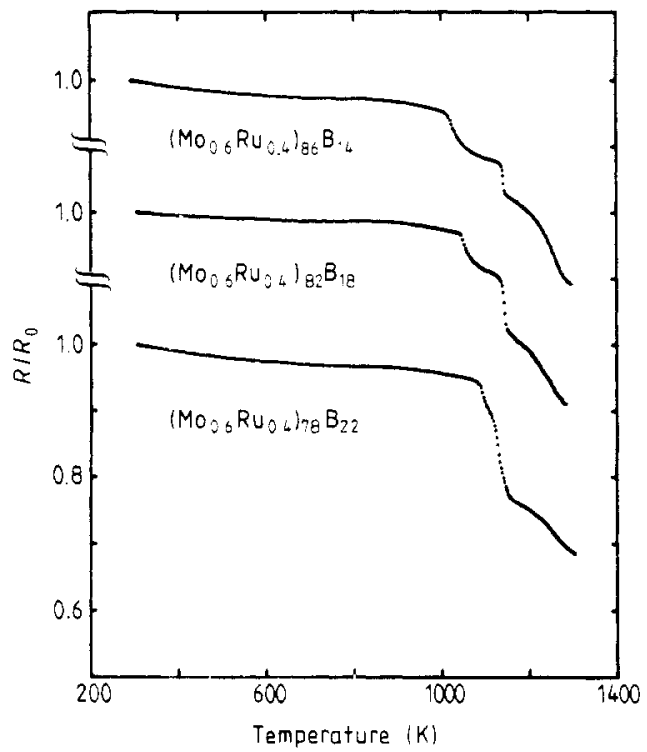

Figure 1. Resistance traces obtained at a heating rate of $15^{\circ} \mathrm{C} \mathrm{min}-1$ for $x=14,18$ and 22 . ( $R_{0}=$ resistance at room temperature.)

step for $x=22$. A shoulder is nevertheless distinguishable. Table 1 gives the crystallisation temperatures and the relative percentage drop of the first two steps. It is clear from the data in the table that the first transition around $1020 \mathrm{~K}$ is characteristic of primary crystallisation from glasses with low boron concentration and the second around $1130 \mathrm{~K}$ from glasses with a high boron content. In order to analyse the crystalline phase corresponding to each step, we applied the heat treatment indicated by arrows shown in figure 2 to a foil of $\left(\mathrm{Mo}_{0.6} \mathrm{Ru}_{0.4}\right)_{86} \mathrm{~B}_{14}$. An $\mathrm{x}$-ray scan was taken after each quench. Figure 3 represents the results for quenches from 1075, 1170 and $1300 \mathrm{~K}$. The crystalline phase identified after the first step is the boron-free sigma phase $\mathrm{Mo}_{5} \mathrm{Ru}_{3}$. During the second step an HCP solid solution of Mo in Ru appears in the structure and from the peak position we estimate the Mo concentration at about $36 \%$. In the final step the Ru HCP phase grows, the sigma phase disappears almost completely and an FCC boride of lattice parameter $a=7.81 \AA$ can be identified.

Thus in glasses of low boron concentration, there is first primary crystallisation of the sigma phase, $\mathrm{Mo}_{5} \mathrm{Ru}_{3}$, which transforms at higher temperature into an HCP Ru. The remaining amorphous state crystallises into a boride phase. In a high boron concentration glass, crystallisation takes place by firstly formation of an HCP solid solution of Mo in Ru and then crystallisation of an FCC boride.

Table 1. Crystallisation temperatures and the percentage drop of the resistivity during the first two steps of the crystallisation.

\begin{tabular}{lllll}
\hline Alloy & $T 1(\mathrm{~K})$ & $\%$ drop & $T 2(\mathrm{~K})$ & $\%$ drop \\
\hline$x=14$ & 1020 & 60 & 1135 & 40 \\
$x=18$ & 1045 & 45 & 1135 & 55 \\
$x=22$ & 1085 & 27 & 1120 & 73 \\
\hline
\end{tabular}




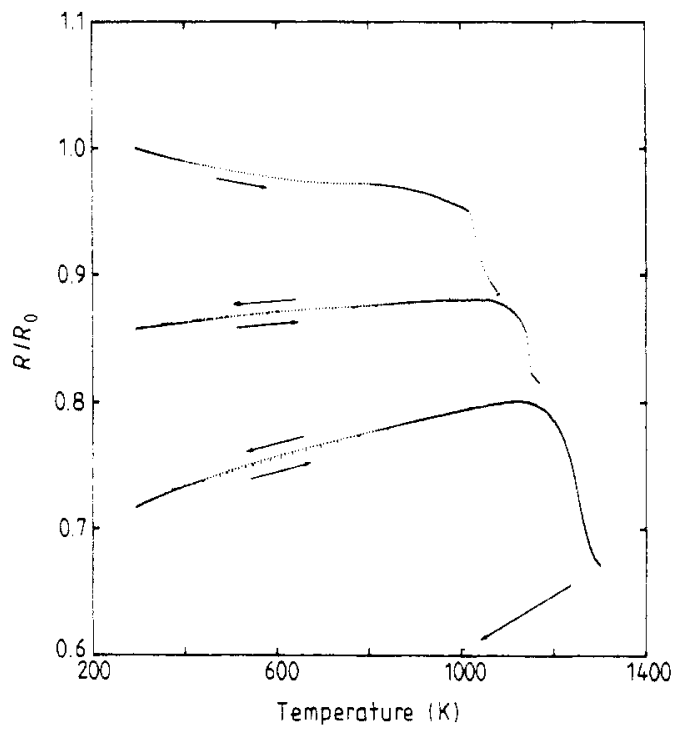

Figure 2. The heat treatment monitored by resistance changes applied to $\left(\mathrm{Mo}_{0.6} \mathrm{Ru}_{0.4}\right)_{86} \mathrm{~B}_{14}$ for the crystallisation study.

As mentioned earlier in the case of $\left(\mathrm{Mo}_{0.6} \mathrm{Ru}_{0.4}\right)_{78} \mathrm{~B}_{22}$ the first two transitions occur in a broad single step. However, if we anneal the sample for $12 \mathrm{~h}$ at $500{ }^{\circ} \mathrm{C}$ we recover a well defined two-step process typical of crystallisation of low boron phases (figure 4). This is what we would expect if annealing induced phase separation by diffusion of boron in the amorphous matrix.

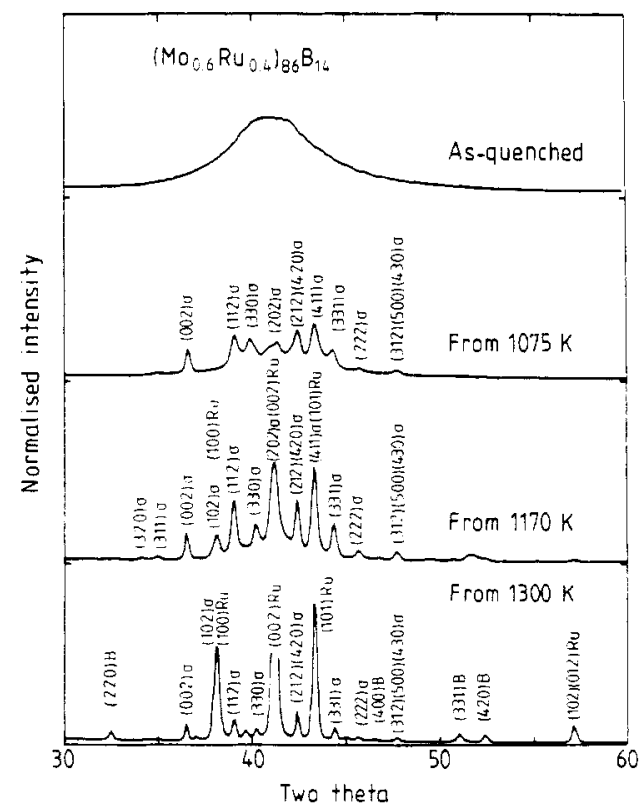

Figure 3. X-ray scan ( $\mathrm{CuK} \mathrm{K}_{a}$ radiation) of the crystalline phases obtained after each quench shown in figure 2. 


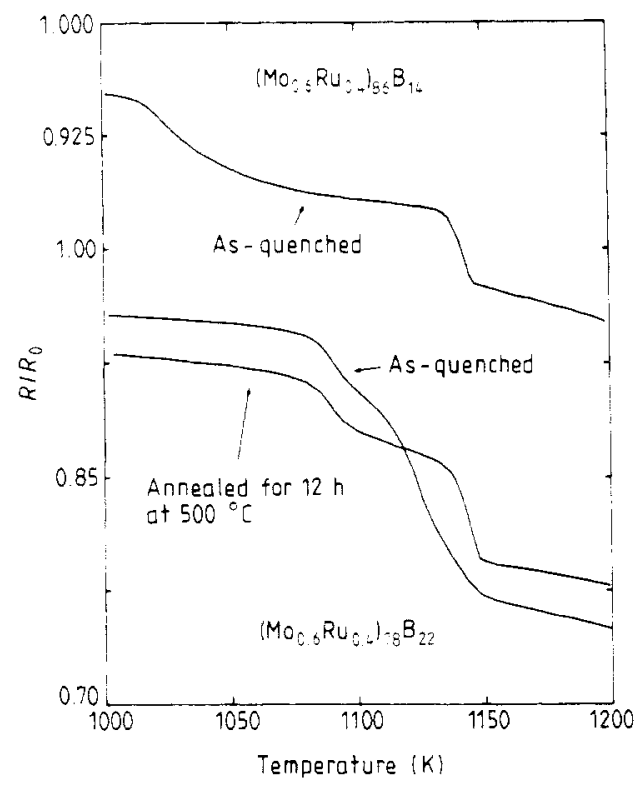

Figure 4. Enlargement of the resistance traces around the crystallisation temperature.

In order to acquire more information about the structural transformation which takes place during annealing, we performed $\mathrm{x}$-ray diffraction measurements on a $\left(\mathrm{Mo}_{0.6} \mathrm{Ru}_{0.4}\right)_{78} \mathrm{~B}_{22}$ sample. All the measurements were made on the same foil in order to avoid fluctuation from sample to sample which might overdraw the rather small change observed during annealing. Figure $5(a)$ shows the radial distribution function in the asquenched state and after annealing for 2 and $11 \mathrm{~h}$ at $525^{\circ} \mathrm{C}$. Table 2 lists the parameters obtained from the $G(R)$. Figure $5(b)$ shows an enlargement of the first three peaks and the $\Delta G(R)=G(R)_{\text {annealed }}-G(R)_{\text {as-quenched }}$ after different heat treatments. Even if $85 \%$ of the total resistivity change occurs during the first two hours [17], only small changes (of the order of $3 \%$ at the main peak) can be observed in the radial distribution function. From table 2 we also note that the nearest-neighbour distance remains unchanged after annealing for two hours. The sizes of these fluctuations are typical of topological relaxation phenomena [24] and they are at the limit of resolution of this experiment. After $11 \mathrm{~h}$, however, we observe a net increase in the nearest-neighbour distance from 2.76 to $2.79 \AA$. We recall that the nearest-neighbour distance in a glass with low boron content $(x=14)$ is $2.83 \AA$. Changes larger than $15 \%$ in the main and second peaks are then taking place. An unusually large increase, by an amount close to $40 \%$, occurs in the shoulder of the second peak at a position which corresponds to the location of the same shoulder in a glass with 14 at.\% boron [17]. The arrows in figure $5(b)$ indicate the position of this shoulder for an as-quenched glass with 14 at.\% boron $\left(R_{2}^{\text {sh }}: 5.58 \AA\right)$ and 22 at.\% boron $\left(R_{2}^{\text {sh }}: 5.38 \AA\right)$. In general, during topological relaxation which results from free-volume annihilation, the peaks become higher and the valleys become deeper. In particular, the split in the second band is enhanced by annealing [24]. In our case, the second peak tends to split into two subpeaks located around $R_{2}^{\prime}=4.3 \AA$ and $R_{2}^{\prime \prime}=4.8 \AA$, as indicated by the bracket in figure $5(b)$. These changes are unusual for free-volume relaxation alone and indicate that some chemical changes are probably also taking place. In fact, these results, together with 

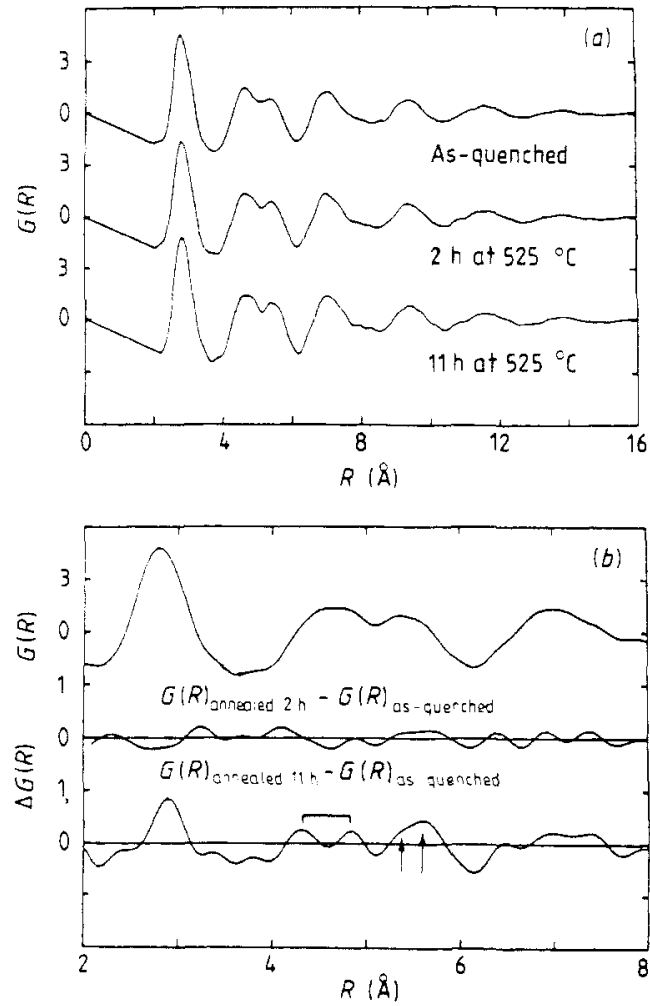

Figure 5. (a) Reduced radial distribution function for $\left(\mathrm{Mo}_{0.6} \mathrm{Ru}_{0.4}\right)_{78} \mathrm{~B}_{22}$ annealed for different times at $525^{\circ} \mathrm{C}$ and $(b)$ the $\Delta G(R)=G(R)_{\text {annealed }}-G(R)_{\text {as-quenched }}$ after different heat treatments at $525^{\circ} \mathrm{C}$.

the resistivity measurements shown in figure 4 strongly suggest the formation of a phase with low boron concentration during annealing.

Figure 6 shows an enlargement of the resistivity scans just before crystallisation. Up to around $600 \mathrm{~K}$ the resistivity varies almost linearly with $T$ as it is found in many metallic glasses at high temperatures $\left(T>\theta_{\mathrm{D}}\right)$ and as predicted by the Ziman-Faber theory in the Debye approximation $[25,26]$. Around $800 \mathrm{~K}$, in region ' $\mathrm{A}$ ' on the plot, a plateau or a departure from the linear temperature dependence is observed. Then the resistivity falls again first slowly in region ' $\mathrm{B}$ ' then rapidly as crystallisation proceeds. Koch et al [16] studied the small-angle $\mathrm{x}$-ray scattering on $\left(\mathrm{Mo}_{0.6} \mathrm{Ru}_{0.4}\right)_{82} \mathrm{~B}_{18}$ and found three regions of interest (figure 10 in reference [16]). For low-temperature annealing $\left(<450^{\circ} \mathrm{C}\right.$ ) little difference is observed in the scattering intensity from that of the as-cast condition. For

Table 2. $G(R)$ parameters.

\begin{tabular}{llllll}
\hline Alloy & Heat treatment & $R 1$ & $R 2$ & $R 2 / R \mathrm{I}$ & $\mathrm{CN}$ \\
\hline $\mathrm{x}=22$ & As quenched & 2.76 & 4.61 & 1.67 & 14.1 \\
& $2 \mathrm{~h}$ & 2.76 & 4.57 & 1.66 & 13.8 \\
& $11 \mathrm{~h}$ & 2.79 & 4.63 & 1.66 & 14.0 \\
\hline
\end{tabular}




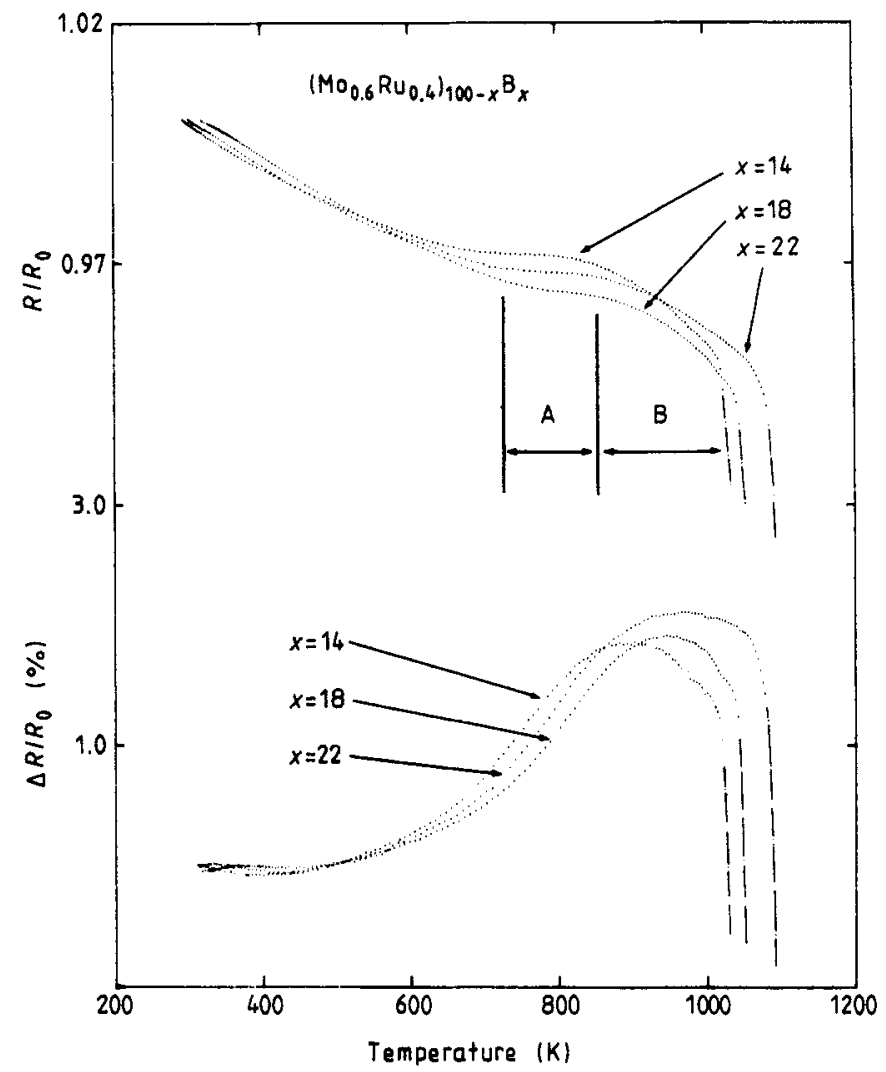

Figure 6. Enlargement of the resistance traces below the crystallisation temperature for $x=14.18$ and 22 . Regions ' $A$ ' and ' $B$ ' correspond to regions II and III in figure 10 of reference [16]. The lower plot represents the excess resistivity above the linear temperature dependence characteristic of the low-temperature region.

higher annealing temperatures or longer times (region II in [16]) peaks in the $I(K)$ curves which can be assumed to represent a 'pseudo Bragg peak' attributed to a periodicity in the scattering entity are observed. For the duration of a resistivity scan, typically two hours, this region corresponds to the plateau ' $\mathrm{A}$ ' in figure 6 . After still higher annealing temperatures or longer times (region III in reference [16]) the peaks in $I(K)$ disappear and the curves can be fitted to the Guinier and Porod law of discrete scatterers. Some crystalline precipitates can be observed by TEM in this region of the temperature-time diagram which corresponds to region 'B' on our resistivity scan. The lower portion of figure 6 shows the excess resistivity above the linear temperature dependence due to thermal scattering. The increase in the excess resistivity seems then related to spinodal decomposition in the amorphous matrix caused by boron diffusion, and the decrease to the onset of crystallisation. Such a maximum in the excess resistivity is well known in the crystalline state. Mimault et al [15] explain the connection between the anomalous initial increase of the electrical resistivity and the presence of a peak in the $\mathrm{x}$-ray small-angle scattering for an $\mathrm{Al}-\mathrm{Zn}$ system when quenched into the spinodal region. Recently, Schulz et al [11] found similar behaviour in the amorphous state during phase separation of a $\mathrm{Cu}_{50} \mathrm{Zr}_{50}$ metallic glass. For $\mathrm{Cu}-\mathrm{Zr}$ the increase of resistivity due to topological relaxation 


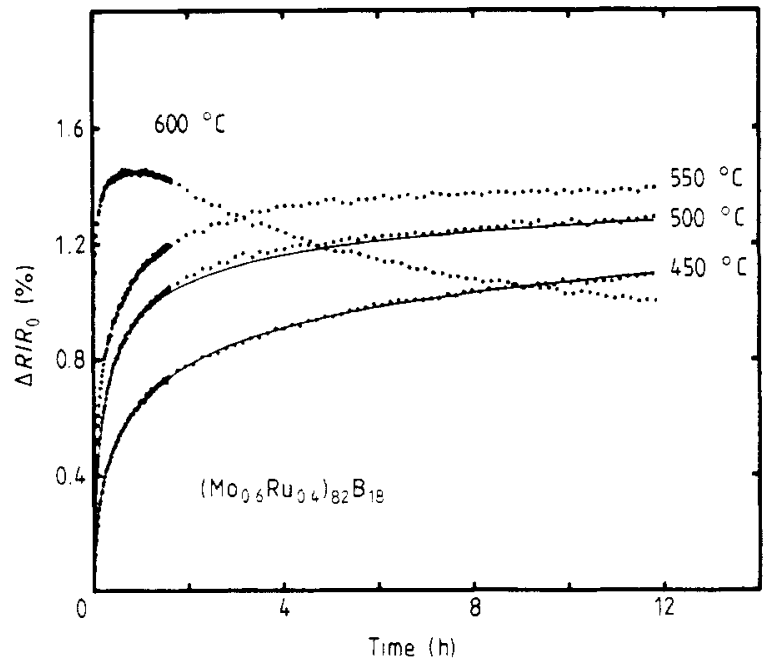

Figure 7. Percentage change in resistance as a function of annealing time at various temperatures. The full curves are fitted to the Kelton-Spaepen theory.

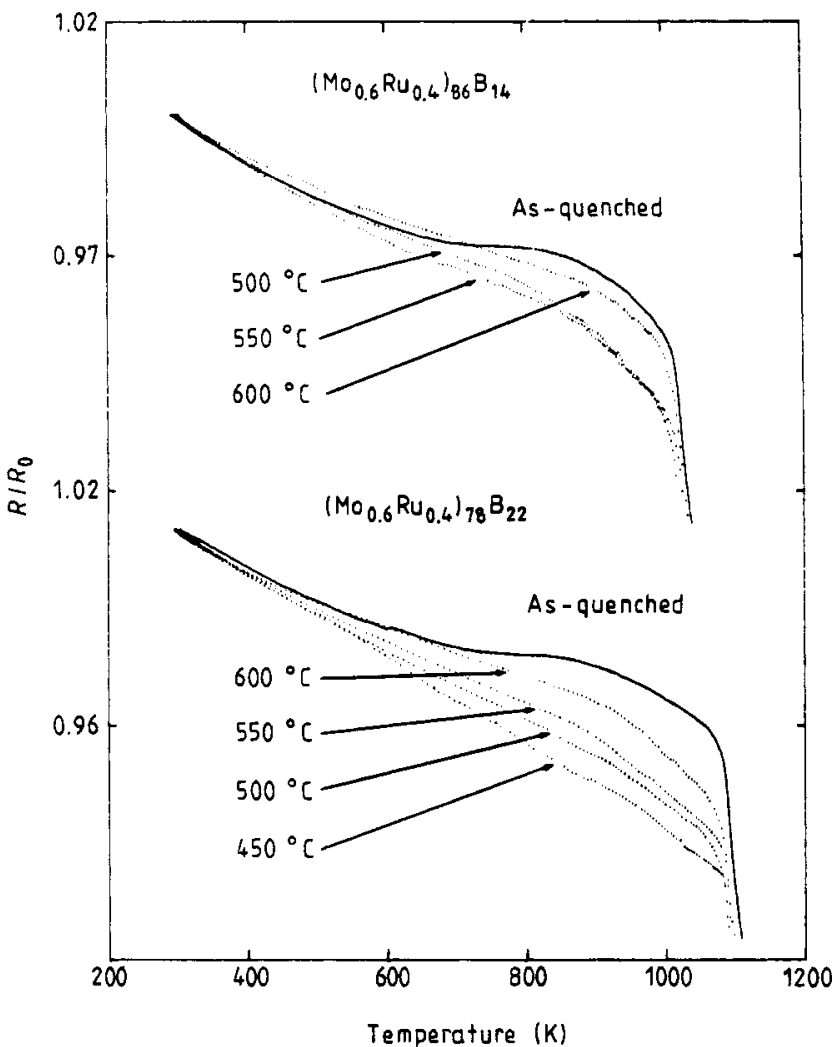

Figure 8. Enlargement of the resistance traces below the crystallisation temperature for $x=14$ and 22 annealed previously for $12 \mathrm{~h}$ at various temperatures. 
and the increase due to chemical segregation are well separated in time during isothermal measurements. This is not the case for $\left(\mathrm{Mo}_{0.6} \mathrm{Ru}_{0.4}\right)_{82} \mathrm{~B}_{18}$ as we can see in figure 7 . At low temperatures $\left(\leqslant 450^{\circ} \mathrm{C}\right.$ ) only topological relaxation (free-volume annihilation) is observed. The full curves represent the best fit of the data using the Kelton-Spaepen model for topological relaxation [7]. At higher temperatures both topological and chemical relaxation contribute to increase the resistivity over about the same time interval. The decrease of the resistivity at $600{ }^{\circ} \mathrm{C}$ after two hours is due to the onset of crystallisation and coincides with the boundary between region II and region III in figure 10 of reference [16]. Note that an increase of the electrical resistivity has been also reported during isochronal annealing of amorphous $\left(\mathrm{Mo}_{0.6} \mathrm{Ru}_{0.4}\right)_{82} \mathrm{~B}_{18}$ films [18].

From figure 6 it seems that there is no correlation between the excess resistivity observed during a scan and the boron concentration of the glass. Figure 8 , however, shows that this anomalous behaviour of the resistivity is very sensitive to the state of relaxation and that annealing has a slightly different effect on boron-14 and boron-22 glasses. If spinodal decomposition is responsible for this resistivity anomaly, topological relaxation or free-volume annihilation should slow down the diffusion [27] of boron and thus affect the behaviour. Johnson and Williams [23] have calculated the effective volume of boron $\bar{V}_{\mathrm{b}}$ across the concentration range. They found that it decreased linearly with increasing boron concentration up to a value close to the effective volume of boron in a close-packed metallic arrangement $V_{\mathrm{b}}^{0}$ near the upper limit of compositions for which a glass can be made (i.e. $x_{\mathrm{m}}=24$ ). If we consider $\bar{V}_{\mathrm{b}}-V_{\mathrm{b}}^{0}$ as a structural excess volume, we can write an effective free volume associated with the boron atom in terms of a thermal free volume and this structural term [17]:

$$
V_{\mathrm{f}}=\alpha\left(T_{\mathrm{f}}-T_{0}\right) \Omega+\beta\left(x_{\mathrm{m}}-x\right) \Omega \quad x<x_{\mathrm{m}} .
$$

$T_{\mathrm{f}}$ is the fictive temperature associated with the quench and $T_{0}$ an appropriate reference temperature at which the thermal free volume is effectively zero. $\Omega$ is the average atomic volume and $\alpha$ and $\beta$ are constants. The diffusion of boron in the matrix depends on this free volume. In the as-quenched condition the thermal term is large and masks any effect of the structural component on the excess resistivity (figure 6). If we annealed out the free volume at low temperature, however, we can reduce the thermal term to $\alpha\left(T_{\mathrm{a}}-T_{0}\right) \Omega$, where $T_{\mathrm{a}}$ is the annealing temperature, and thus observe the effect of the concentration on the resistivity bump. Figure 8 shows that in the case of $\left(\mathrm{Mo}_{0.6} \mathrm{Ru}_{0.4}\right)_{78} \mathrm{~B}_{22}$, for which the structural component vanishes $\left(x \approx x_{\mathrm{m}}\right)$, the resistivity anomaly completely disappears after annealing for $12 \mathrm{~h}$ at $450^{\circ} \mathrm{C}$. The effective boron free volume vanishes preventing its diffusion, and the resistivity varies linearly all the way up to the crystallisation temperature. For higher annealing temperatures followed by a quench to room temperature we observe the gradual effect of the increase in the quenched-in thermal free volume. The resistivity anomaly becomes more significant at higher annealing temperatures. For $\left(\mathrm{Mo}_{0.6} \mathrm{Ru}_{0.4}\right)_{86} \mathrm{~B}_{14}$ the structural component is not negligible $\left(x \ll x_{\mathrm{m}}\right)$ and low-temperature annealing is not sufficient to stop the diffusion of boron.

Unlike in the crystalline state, the very short electronic mean free path ( $3-4 \AA)$ in these glasses casts doubt on an approach using the Ziman scattering theory for resistivity and small-angle $x$-ray scattering data from spinodal decomposition theory to explain theoretically the resistivity anomaly observed here. Figure 9 compares the excess resistivity with the change in the electronic density of states in terms of the reduced annealing temperature. $T_{\mathrm{cr}}$ is the crystallisation temperature corresponding to the onset of the drastic drop in the measured property. We see that the maximum in the resistivity corresponds to a minimum in the density of states. Based on a similar correlation between the fractional 


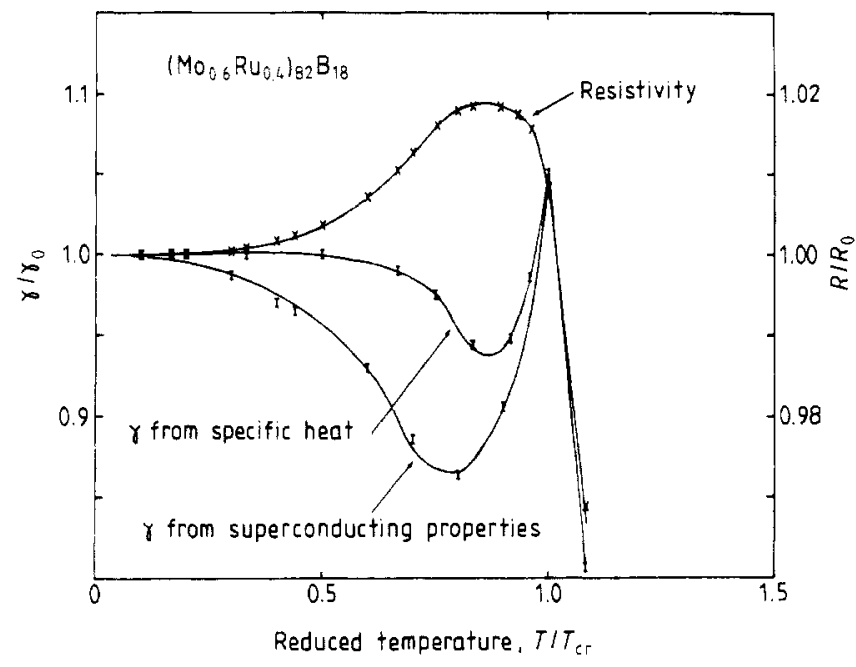

Figure 9. Correlation between the excess resistivity and the change in the density of states (from Koch et al [16]) against the reduced annealing temperature. $T_{\mathrm{cr}}$ is the crystallisation temperature $\left({ }^{\circ} \mathrm{C}\right)$ corresponding to the onset of the drastic drop in the measured property.

change in magnetic susceptibility and the change in resistivity upon crystallisation, Altounian et al [28] suggest that $\mathrm{s}-\mathrm{d}$ scattering, which is proportional to the density of states, makes an important contribution to the resistivity for $\mathrm{Cu}-\mathrm{Zr}$ and $\mathrm{Ni}-\mathrm{Zr}$ glasses. Our results on $\mathrm{Mo}-\mathrm{Ru}-\mathrm{B}$ alloys show that even if the resistivity and density of states are correlated during crystallisation they behave in opposite directions in the amorphous state. Recently, Ballentine et al [29] have calculated the conductivities of several liquid transition metals characterised by strong scattering and $s-d$ hybridisation using the Kubo formula and clusters of 365 atoms whose pair distribution functions agree with the measured values. They treated the $s$ and $d$ states on an equal footing in order to evaluate the relative contributions of the $s$ and $d$ states to the conductivity. They found that although the diffusivity of $d$ states is less than that of $s$ states, the $d$ states dominate conductivity because of their much greater density. They wrote [29]

$$
\sigma=\left(e^{2} / \Omega\right)\left(D_{\mathrm{s}} N_{\mathrm{s}}\left(\varepsilon_{\mathrm{F}}\right)+D_{\mathrm{d}} N_{\mathrm{d}}\left(\varepsilon_{\mathrm{F}}\right)\right)
$$

where $N_{\mathrm{s}}$ and $N_{\mathrm{d}}$ are the $\mathrm{s}$ and d partial densities of states and $D_{\mathrm{s}}$ and $D_{\mathrm{d}}$ the respective diffusivities. The sd contribution to the conductivity was found to be negligible. For liquid $\mathrm{La}, \mathrm{Cr}, \mathrm{Mn}$ and $\mathrm{Fe}$ the $\mathrm{d}$ states contribute about $85 \%$ of the conductivity.

There are several reasons to believe that this is also the case for the $\left(\mathrm{Mo}_{0.6} \mathrm{Ru}_{0.4}\right)_{100-x} \mathrm{~B}_{x}$ glasses. In those cases we can write in a first approximation

$$
\sigma \approx\left(e^{2} / \Omega\right) D_{\mathrm{d}} N_{\mathrm{d}}\left(\varepsilon_{\mathrm{F}}\right)
$$

The average electronic diffusivity $D=\frac{1}{3} v_{\mathrm{F}} l$ (where $v_{\mathrm{F}}$ is the average group velocity at the Fermi surface and $l$ is the average electron mean free path) is related to the critical field gradient by [30]

$$
\mathrm{d} H_{\mathrm{c} 2} /\left.\mathrm{d} T\right|_{T_{\mathrm{c}}}=4 k_{\mathrm{B}} c / \pi e D .
$$

If the electrons involved in the superconductive pairing are also the ones responsible for the 


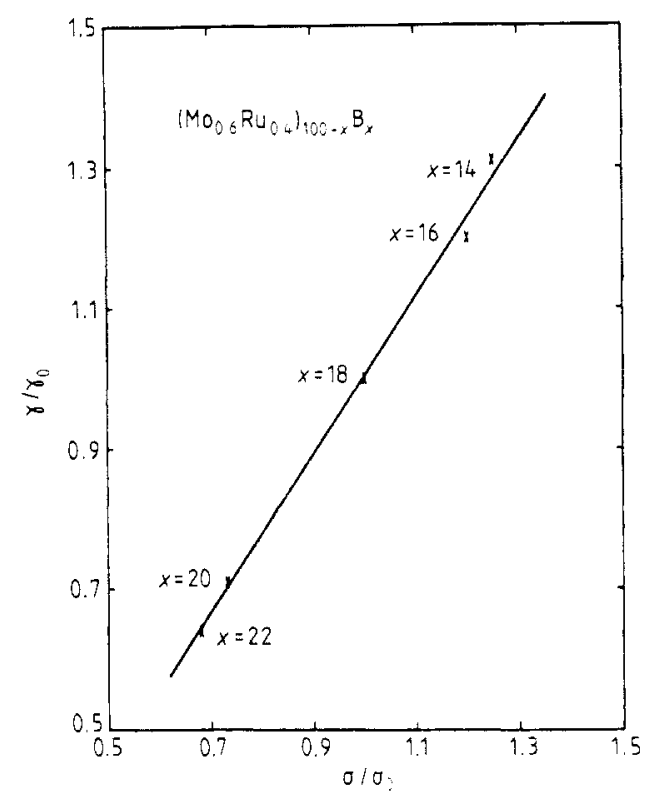

Figure 10. Normalised density of states against conductivity for different alloy concentrations. The data are from Hopkins et al [22] and from Johnson et al [23].

conductivity in the normal state, it follows from (3) and (4) that

$$
N_{\mathrm{d}}\left(\varepsilon_{\mathrm{F}}\right)=\left(\left.\frac{\pi \Omega}{4 e k_{\mathrm{B}} c} \frac{\mathrm{d} H_{\mathrm{c} 2}}{\mathrm{~d} T}\right|_{T_{\mathrm{c}}}\right) \sigma .
$$

It has been shown [23] that for $\left(\mathrm{Mo}_{0.6} \mathrm{Ru}_{0.4}\right)_{100-x} \mathrm{~B}_{x}$ the critical field gradient does not vary with the concentration to within experimental uncertainty. A plot of the density of states against the conductivity for different concentrations would give, therefore, according to equation (5), a straight line and this is what we observe experimentally; see figure 10.

Since the conductivity is directly proportional to the density of $d$ states at the Fermi level, the Mott model which predicts an increase in resistivity with an increase in the density of $d$ states through the $s-d$ scattering mechanism does not seem to be applicable for $\mathrm{Mo}-\mathrm{Ru}-\mathrm{B}$ glasses and the results suggest instead a d-band conduction mechanism. More evidence for d-band conduction in metallic glasses will be published in a separate report [31].

The correct explanation of this resistivity anomaly must then be the following: annealing induces phase separation into boron-rich and boron-poor zones. This spatial variation in the metalloid concentration affects the density of states at the Fermi level $N_{\mathrm{d}}\left(E_{\mathrm{F}}\right)$ and thus the conductivity in a d-band conduction picture.

\section{Conclusions}

(1) Primary crystallisation of a boron-free phase, HCP solid solution of Mo in Ru for high boron concentration glasses and sigma phase $\mathrm{Mo}_{5} \mathrm{Ru}_{3}$, which then decomposes at higher temperatures into the HCP Ru for low boron concentration, is responsible for the 
first two steps in the resistivity drop around the crystallisation temperature. In the final step, the remaining boron-rich amorphous matrix crystallises into an FCC boride.

(2) The anomalous excess resistivity above the usual linear temperature dependence predicted by the Ziman theory at high temperatures is explained in terms of phase separation caused by long-range diffusion of boron, which affects the density of states at the Fermi level and thus the number of carriers in a d-band conduction model.

(3) The changes in the radial distribution function upon annealing at $525^{\circ} \mathrm{C}$ cannot be explained by topological relaxation alone. Some types of chemical relaxation probably caused by phase separation must occur as well.

\section{Acknowledgments}

The authors would like to thank Concetto Geremia for his technical assistance. One of us (RS) is grateful to the National Research Council of Canada for financial assistance. This work was supported by the Department of Energy under Contract No DE-AM03$76 \mathrm{SF} 00767$.

\section{References}

[1] Balanzat M 1980 Scr. Metall. 14173 Balanzat E. Mairy C and Hillairet J 1980 J. Physique 41871

[2] Marcus M 1979 Acta Metall. 27879

[3] Allia P, Sato Turtelli R and Vinai F 1982 Solid State Commun. 43821

[4] Srolovitz D, Egami T and Vitek V 1981 Phys. Rev. B 246936

[5] Allia P, Andreone D, Sato Turtelli R and Vinai F 1982 J. Appl. Phys. 538798

[6] Venkataraman S, Reddy K V. Virataswaroop U N, Rao G V and Bhatnagar A K 1984 J. Non-Cryst. Solids 65423

[7] Kelton K F and Spaepen F 1981 Proc. 4th Int. Conf. on Rapidly Quenched Metals ed. T Masumoto and K Suzuki pp 527-30

[8] Chen C P and Turnbull D 1975 J. Non-Cryst. Solids 17169

[9] Walter J L. Legrand D G and Luborsky F E 1977 Mater. Sci. Eng. 29161

[10] Piller J and Haasen P 1982 Acta Metall. 30 I

[11] Schulz R, Samwer K and Johnson W L 1984 J. Non-Cryst. Solids 61-62 997

[12] Rossiter P L and Wells P 1971 Phil. Mag. 24 425; 1971 J. Phys. C: Solid State Phys. 4354

[13] Hillel A J, Edwards J T and Wilkes P 1975 Phil. Mag. 32 189; 1977 Phil. Mag. 351221

[14] Binder K and Stauffer D 1976 Z. Phys. B 24407

[15] Mimault J, Delafond J, Jungua A, Naudon A and Grille J 1978 Phil. Mag. B 38255

[16] Koch C C, Kroeger D M, Lin J S and Scarbrough J O 1983 Phys. Rev. B 271586

[17] Mehra M, Schulz R and Johnson W L 1984 J. Non-Cryst. Solids 61-62 859

[18] Soldner L. Adrian H and Saemann-Ischenko G 1983 Appl. Phys. Lett. 43788

[19] Pietrokowsky P 1963 Rev. Sci. Instrum. 34445

[20] Williams A 1981 PhD Thesis California Institute of Technology, Pasadena, CA

[21] Cargill G S III 1975 Solid State Phys. 30227 (New York: Academic)

[22] Hopkins S and Johnson W L 1982 Solid State Commun. 43537

[23] Johnson W L and Williams A R 1979 Phys. Rev. B 201640

[24] Egami T 1979 J. Mater. Sci. 141249

[25] Cote P J and Meisel L V 1977 Phys. Rev. Lett. 39102

[26] Nagel S R 1977 Phys. Rev. B 161694

[27] Chen H S. Kimerling L C. Poate J M and Brown W L 1978 Appl. Phys. Lett. 32461

[28] Altounian Z, Guo-hua T and Strom-Olsen J O 1982 J. Appl. Phys. 534755

[29] Ballentine L E, Bose S K and Hammerberg J E 1984 J. Non-Cryst. Solids 61-62 1195

[30] Helfand E and Werthamer N R 1964 Phys. Rev. Lett. 13 686; 1966 Phys. Rev. 147295

[31] Schuiz R and Johnson W L to be published 